\title{
ESTADO DE LAS POBLACIONES DE MAMÍFEROS EN LA RESERVA NACIONAL MATSÉS, AL NORESTE DE LA AMAZONÍA PERUANA
}

\author{
León TORRES-OYARCE ${ }^{1}$, Claudio BARDALES-ALVITES ${ }^{1}$, Emérita TIRADO-HERRERA², Miguel \\ ANTÚNEZ-CORREA ${ }^{3}$ \\ 1 Facultad de Ciencias Biológicas. Universidad Nacional de la Amazonía Peruana. Calle Pevas 5ta cuadra S/N. Iquitos, \\ Loreto-Perú. \\ leonfernandotorresoyarce@gmail.com \\ 2 Departamento de Ecología y Fauna. Facultad de Ciencias Biológicas. Universidad Nacional de la Amazonía Peruana. Calle \\ Pevas 5 ta cuadra S/N. Iquitos, Loreto-Perú. \\ 3 Instituto del Bien Común. Jirón Nauta ํㅜ34. Iquitos, Loreto-Perú
}

\section{RESUMEN}

Este estudio se realizó entre junio a octubre del 2015, en la Reserva Nacional Matsés, con el objetivo de conocer el estado de las poblaciones de mamíferos de caza en tres sectores de muestreo: Alemán, Loboyacu y Alto Gálvez. Los datos fueron colectados en cuatro transectos lineales, cada uno tuvo $5 \mathrm{~km}$ de longitud. En los tres sectores de muestreo, los primates tuvieron las mayores densidades poblacionales. En el sector Alto Gálvez, las especies con mayor densidad fueron Saimiri macrodon (26.75 ind $\left./ \mathrm{km}^{2}\right)$ y Sapajus macrocephalus (18.93 ind $\left./ \mathrm{km}^{2}\right)$; mientras que en los sectores Alemán y Loboyacu, fue Lagothrix lagotricha (23.54 y 13.84 ind $/ \mathrm{km}^{2}$, respectivamente) y Saguinus mystax (20.58 y $17.87 \mathrm{ind} / \mathrm{km}^{2}$, respectivamente). En general, la variabilidad de mamíferos de caza puede ser explicado al $67.52 \%$. Las comunidades de mamíferos de los sectores Alemán y Loboyacu fueron similares, en donde la caza fue frecuente. La comparación independiente de ambos sectores versus el sector del Alto Gálvez, mostró que el último sector tuvo mayor densidades poblacional. Es decir, la cuenca alta del río Gálvez mantiene las poblaciones más saludables de mamíferos.

PALABRAS CLAVE: Transectos, Mamíferos, Densidad, Reserva Nacional Matsés.

\section{STATUS OF MAMMALIAN POPULATIONS IN THE NATIONAL MATSES RESERVE, PERUVIAN NORTHERN AMAZON}

\section{ABSTRACT}

This study was conducted between June and October 2015, in the Matsés National Reserve, with the objective of knowing the status of hunting mammal populations in three sampling sectors: Alemán, Loboyacu and Alto Gálvez. The data were collected in four linear transects, each one had $5 \mathrm{~km}$.

In the three sampling sectors, the primates had the highest population densities. In the Alto Gálvez sector, the species with the highest density were Saimiri macrodon (26.75 ind $/ \mathrm{km}^{2}$ ) and Sapajus macrocephalus (18.93 ind / $\mathrm{km}^{2}$ ); while in the Aleman and Loboyacu sectors, it was Lagothrix lagotricha (23.54 and 13.84 ind $/ \mathrm{km}^{2}$, respectively) and Saguinus mystax (20.58 and $17.87 \mathrm{ind} / \mathrm{km}^{2}$, respectively).

In general, the variability of game mammals can be explained to $67.52 \%$. Mammal communities of the Aleman and Loboyacu sectors were similar, where hunting was frequent. The independent comparison of both sectors versus the Alto Gálvez sector showed that the last sector had higher population densities. That is, the upper basin of the Gálvez River maintains the healthiest populations of mammals.

KEYWORDS: Density, Matses National Reserve, Transects lineal, Mammals. 


\section{INTRODUCCIÓN}

La Reserva Nacional Matsés es un área protegida que alberga una gran diversidad de especies, entre ellas 65 especies de mamíferos de las 292 registradas para la Amazonía peruana (Vriesendorp et al., 2006). Este grupo de vertebrados desempeña un rol importante en la dinámica de los ecosistemas como eficientes dispersores de semillas, polinizadores y controladores biológicos de poblaciones bióticas (Rumiz 2010). Sin embargo, en la actualidad se sabe que diferentes amenazas provenientes de actividades antrópicas van en aumento. Por ello, conocer parámetros poblacionales como la densidad de mamíferos, permitirá detectar cambios poblacionales en el momento oportuno.

En la Amazonía peruana se han realizado diversos estudios para conocer la densidad de mamíferos. Estos estudios fueron realizados en la Reserva Nacional Pacaya-Samiria (Aquino et al., 2001; Aquino \& Calle 2003; Bodmer et al., 2014), Área de Conservación Regional Comunal Tamshiyacu-Tahuayo (Bodmer et al., 1997; Lavajos \& Meza 2013), Área de Conservación Regional Ampiyacu-Apayacu (Puertas et al., 2017), Reserva Nacional Pucacuro (Aquino et al., 1997; Pérez-Peña et al., 2012), río Algodón (Aquino et al., 2007a), río Itaya (Aquino et al., 2007b), Lago Preto Paredón (Pérez-Peña, 2011), río Curaray (Aquino et al., 2015), Alto Purús (Lleellish et al., 2003), ríos bajo Urubamba y Tambo (Aquino et al., 2014). Cada uno de los estudios mencionados resalta el estado poblacional de las especies, y su contribución en las decisiones del manejo y conservación de los mamíferos en las zonas mencionadas.
En la Reserva Nacional Matsés, la caza practicada por la población local con fines de subsistencia, está siendo monitoreada por la jefatura de la reserva. En tal sentido, es de importancia el conocimiento del estado actual de las poblaciones de mamíferos, mediante la obtención de información base para realizar el monitoreo de las especies. Esto, sumado al conocimiento de la presión de caza de las especies, permitirá recomendar el aprovechamiento de las especies cuya caza estaría siendo sostenible. De esta manera se estaría contribuyendo en la generación de documentos de gestión que permitan obtener beneficios económicos y de subsistencia a las comunidades Matsés, mediante prácticas que sean congruentes con la conservación de los bosques de esta importante área natural protegida.

\section{MATERIALES Y MÉTODOS}

\section{ÁREA DE ESTUDIO}

El estudio se realizó en los sectores Alemán $\left(5^{\circ} 25^{\prime} 22.40^{\prime \prime} \mathrm{S}, 73^{\circ} 46^{\prime} 50.55^{\prime \prime O}\right)$ Loboyacu ( $\left.5^{\circ} 15^{\prime} 40.54^{\prime \prime S}, 73^{\circ} 24^{\prime} 33.93^{\prime \prime O}\right)$ y Alto Río Gálvez $\left(5^{\circ} 38^{\prime} 47.00^{\prime \prime} \mathrm{S}, 73^{\circ} 34^{\prime} 14.39^{\prime \prime} \mathrm{O}\right)$ pertenecientes a la Reserva Nacional Matsés (RNM) que cuenta con una superficie de 420,635.34 ha,ubicada en la región Loreto, al noreste de la Amazonía peruana, a $150 \mathrm{~km}$ al sureste de la ciudad de Iquitos. La RNM se encuentra en los distritos de Requena, Soplin y Yaquerana, y en la provincia de Requena de la Región Loreto. Geográficamente se ubica entre las cuencas de los ríos Yavarí y el bajo Ucayali, entre el interfluvio de los ríos Tapiche, Blanco, Gálvez y Yaquerana, limitando al este con las cabeceras del

Tabla 1. Coordenadas UTM de los transectos de muestreo por sectores.

\begin{tabular}{cccccc}
\hline \multirow{2}{*}{ Sector } & \multirow{2}{*}{ Transecto } & \multicolumn{2}{c}{ Coordenada Inicial } & \multicolumn{2}{c}{ Coordenada Final } \\
\cline { 2 - 5 } & & $\mathbf{X}$ & $\mathbf{Y}$ & $\mathbf{X}$ & $\mathbf{Y}$ \\
\hline \multirow{3}{*}{ Alemán } & TrA_01 & 635255 & 9401222 & 637927 & 9404734 \\
& TrA_02 & 634969 & 9399782 & 636541 & 9395166 \\
& TrA_03 & 640246 & 9384000 & 650345 & 9382922 \\
& TrA_04 & 640345 & 9382922 & 644299 & 9379930 \\
\hline \multirow{3}{*}{ Loboyacu } & TrL_01 & 675759 & 9418296 & 672253 & 9414474 \\
& TrL_02 & 676750 & 9418200 & 680477 & 9414834 \\
& TrL_03 & 680577 & 9402388 & 680600 & 9407180 \\
& TrL_04 & 679473 & 9402136 & 674483 & 9401835 \\
\hline \multirow{3}{*}{ Alto Gálvez } & TrG_01 & 666005 & 9383328 & 661020 & 9383328 \\
& TrG_02 & 666794 & 9384512 & 666704 & 9389489 \\
& TrG_03 & 658507 & 9376207 & 656813 & 9380879 \\
& TrG_04 & 658000 & 9375300 & 654072 & 9372200 \\
\hline
\end{tabular}




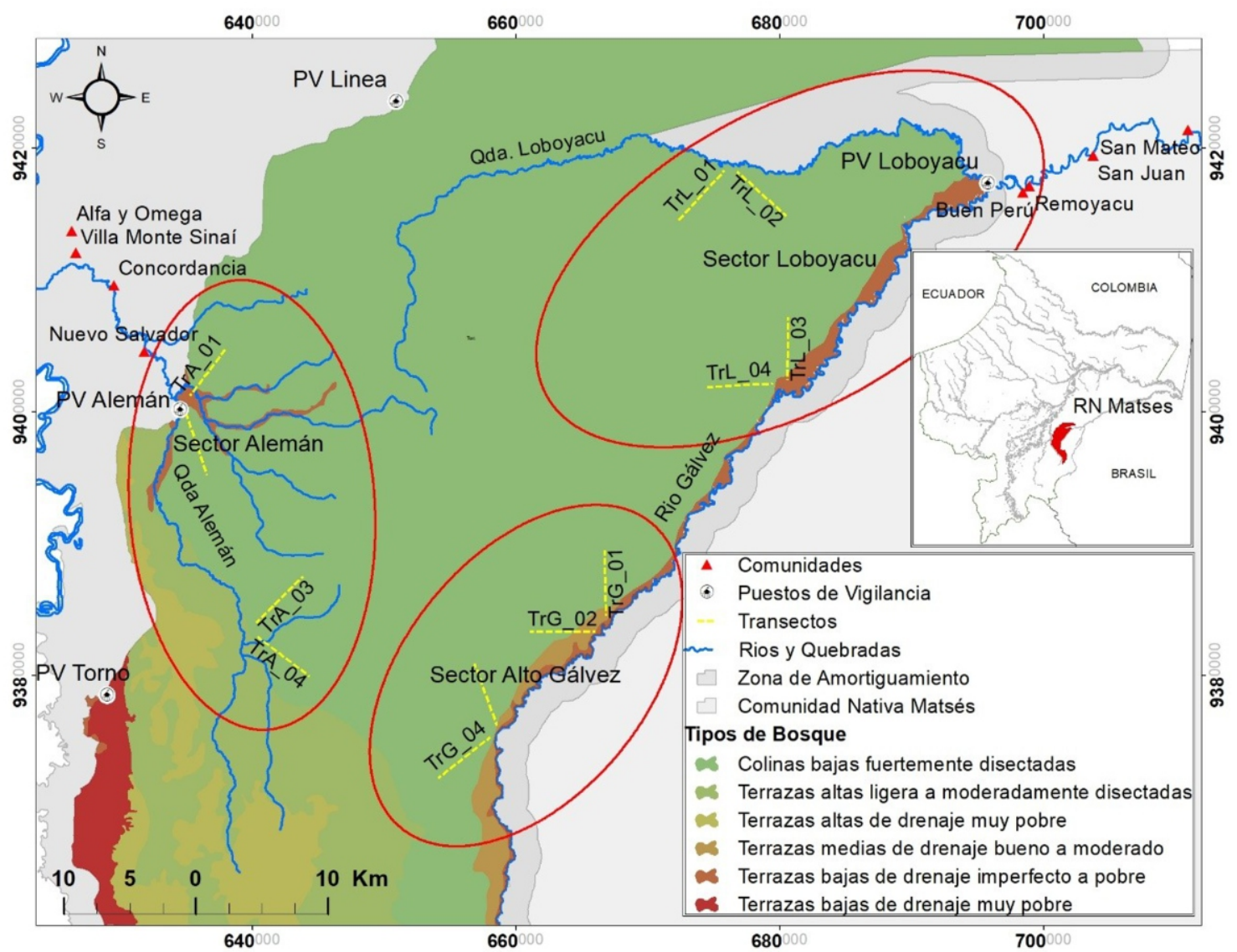

Figura 1. Mapa de registros de Spizaetus melanoleucus en la Reserva Nacional Allpahuayo Mishana y río Sucusari, Loreto, Perú.

río Gálvez y el oeste a unos $3 \mathrm{~km}$ del río Blanco. $\mathrm{Al}$ sur colinda con el Parque Nacional Sierra del Divisor, al este con la Comunidad Nativa Matsés (SERNANP, 2014a).

El sector Alemán, comprende ambas márgenes de la quebrada Alemán, que es un afluente del río Tapiche, abarcando bosques de colinas bajas fuertemente disectadas, terrazas altas de ligera a moderadamente disectadas, terrazas altas de drenaje muy pobre y terrazas bajas de drenaje imperfecto a pobre. Mientras que el sector Loboyacu se ubica margen derecha de la quebrada Loboyacu y la margen izquierda de la parte media del río Gálvez, ocupando bosques de colinas bajas fuertemente disectadas y terrazas bajas de drenaje imperfecto a pobre. Finalmente, el sector Alto Gálvez comprende la margen izquierda de la parte alta del río Gálvez, abarcando bosques de colinas bajas fuertemente disectadas, terrazas medias de drenaje bueno a moderado y terrazas bajas de drenaje imperfecto a pobre.

\section{MÉTODOS}

\section{Técnica de muestreos por transectos lineales}

Los muestreos se realizaron durante la época de vaciante entre los meses de junio y octubre del 2015. Para ello, se abrieron transectos con un mínimo de 24 horas de anticipación a los muestreos. En el presente estudio, las trochas de los cazadores fueron excluidas a fin de evitar áreas que estén sujetas a la frecuente perturbación humana. Cada sector contó con dos estaciones de muestreos y en cada estación se abrieron dos transectos de $5 \mathrm{~km}$ de longitud (Figura 1 y Tabla 1), los cuales fueron recorridos ida y vuelta, desde las 7:00 hasta las 16:00 horas. El desplazamiento por los transectos se realizó a una velocidad promedio de $1 \mathrm{~km} /$ hora, con paradas para escuchar a los animales. Cada vez que hubo un avistamiento se procedió a registrar la distancia perpendicular del primer individuo avistado al transecto y el número total de individuos del grupo, además de la hora, hábitat y ubicación en el transecto. 


\section{Análisis de datos}

Para aquellas especies con igual o superior a los 15 avistamientos, se estimó la densidad mediante el software DISTANCE 6.2, cuya fórmula es la siguiente:

$$
D=\frac{N f(0)}{2 L}
$$

Donde:

$$
\begin{aligned}
\mathrm{D}= & \text { Densidad. } \\
\mathrm{N}= & \text { Número de animales avistados. } \\
\mathrm{F}(\mathrm{o})= & \text { Función de probabilidad de avistar los } \\
& \text { animales. } \\
\mathrm{L}= & \text { Longitud del recorrido } \\
2 \quad= & \text { Constante que indica que el muestreo se } \\
& \text { realiza a ambos lados del transecto. }
\end{aligned}
$$

Para las especies con avistamientos menores a 15, la densidad se estimó usando la fórmula de ancho fijo (Burnham et al., 1980):

$$
D=\frac{N}{2 A L}
$$

Donde:

$$
\begin{aligned}
\mathrm{D}= & \text { Densidad. } \\
\mathrm{N}= & \text { Número de animales avistados dentro del } \\
& \text { ancho fijo. } \\
\mathrm{A}= & \text { Ancho propuesto de la faja del hábitat que } \\
& \text { es cubierto. } \\
\mathrm{L}= & \text { Longitud del transecto. } \\
2= & \begin{array}{l}
\text { Constante que indica que el muestreo se } \\
\text { realiza en ambos lados del transecto. }
\end{array}
\end{aligned}
$$

Las especies que determinan más variabilidad de los sectores fueron conocidas mediante el Análisis de Componentes Principales, asimismo se realizó la prueba no paramétrica de ANOSIM para conocer las diferencias de las comunidades de mamíferos entre los sectores de estudio.

\section{RESULTADOS}

En los sectores evaluados de la Reserva Nacional Matsés, se recorrió un total de $674.4 \mathrm{~km}$, y se tuvo 399 registros de 20 especies, distribuidas en 7 órdenes. En el sector Alto Gálvez se registraron 18 especies, mientras que el sector Alemán y Loboyacu tuvieron 16 y 15 especies, respectivamente. En los tres sectores, el orden primate tuvo mayor cantidad de especies.

Los primates tuvieron las más altas densidades en los tres sectores del estudio. En el sector Alemán, las densidades más altas fueron de Lagothrix lagotricha $\left(23.54 \mathrm{ind} / \mathrm{km}^{2}\right)$, Saguinus mystax (20.58 ind $\left./ \mathrm{km}^{2}\right)$, Saguinus nigrifrons $\left(10.50 \mathrm{ind} / \mathrm{km}^{2}\right)$ y Pithecia monachus $\left(9.68 \mathrm{ind} / \mathrm{km}^{2}\right)$. Mientras que,
Tapirus terrestris $\left(0.11 \mathrm{ind} / \mathrm{km}^{2}\right)$ y Choloepus didactylus $\left(0.11 \mathrm{ind} / \mathrm{km}^{2}\right)$ tuvieron las densidades más bajas, este ultimo fue únicamente registrado en este sector, pero no se registraron a Ateles chamek, Tamandua tetradactyla, Tayassu pecari y Myoprocta pratti. En Loboyacu, las mayores densidades correspondieron a Saguinus mystax (17.87 ind $\left./ \mathrm{km}^{2}\right)$, Lagothrix lagotricha (13.85 ind $\left./ \mathrm{km}^{2}\right)$, Saguinus nigrifrons $\left(10.88 \mathrm{ind} / \mathrm{km}^{2}\right)$, Sapajus macrocephalus $\left(10.37 \mathrm{ind} / \mathrm{km}^{2}\right)$ y Pithecia monachus ( $\left.9.12 \mathrm{ind} / \mathrm{km}^{2}\right)$, y las densidades más bajas fueron de Eira barbara (1.12 ind $\left./ \mathrm{km}^{2}\right)$, Tamandua tetradactyla (1.12 ind $\left./ \mathrm{km}^{2}\right)$ y Myoprocta pratti $(0.16$ ind $/ \mathrm{km}^{2}$ ) que fue únicamente reportada en este sector. Además, no hubieron registros de Alouatta seniculus, Saimiri macrodon, Nasua nasua, Tayassu pecari y Choloepus didactylus. En el alto Gálvez, las densidades más altas fueron de Saimiri macrodon (26.75 ind $\left./ \mathrm{km}^{2}\right)$, S. macrocephalus $\left(18.94 \mathrm{ind} / \mathrm{km}^{2}\right)$, L. lagotricha $\left(15.04 \mathrm{ind} / \mathrm{km}^{2}\right), \quad$ S. mystax $(13.23$ ind $\left./ \mathrm{km}^{2}\right)$, A. chamek $\left(9.58 \mathrm{ind} / \mathrm{km}^{2}\right)$, y las densidades más bajas fueron de T. terrestris $\left(0.34 .12 \mathrm{ind} / \mathrm{km}^{2}\right)$, Eira barbara $\left(0.34 \mathrm{ind} / \mathrm{km}^{2}\right)$ y $T$. tetradactyla $(0.34$ ind $\left./ \mathrm{km}^{2}\right)$. Tayassu pecari estuvo presente en este sector (Tabla 2).

La variabilidad de mamíferos de caza entre los sectores puede ser explicada al $64.65 \%$ en dos dimensiones o componentes principales. El componente principal I explica que hay una variabilidad del $39.14 \%$, y las especies más importantes que explican esta variabilidad fueron $S$. macrodon (0.74) y Tayasssu tajacu (0.29). Mientras que en el componente principal II explica una variabilidad del $25.51 \%$, las especies que ayudan a explicar fueron $S$. nigrifrons $(0.38)$ y $L$. lagotricha (0.22). En general, las comunidades de mamíferos en el sector Alemán y Loboyacu fueron parecidas $(\mathrm{P}=0.59)$, donde la caza está siendo frecuente, mientras que al comparar estos sectores con el Alto Gálvez, las comunidades de mamíferos resultaron diferentes $(\mathrm{P}=0.01)$.

\section{DISCUSIÓN}

En cada uno de los sectores en estudio, los primates tuvieron altas densidades, siendo los primates de tamaño pequeño los más abundantes. Este patrón de abundancia se refleja en estudios realizados en otros lugares de la Amazonía peruana, como en la cuenca alta del río Itaya (Aquino et al., 2007a), río Algodón (Aquino et al., 2007b), Reserva Nacional Pucacuro (Pérez-Peña et al., 2012), Área de Conservación Regional Tamshiyacu-Tahuayo (Lavajos \& Meza 2013), cuyas densidades indicarían que las especies pequeñas de primates se adaptan a los ambientes impactados y/o abandonados por primates grandes a causa de actividades antrópicas (Aquino et al., 2007a; Rosin \& Swamy 2013). 
Tabla 2. Densidades de mamíferos de caza de tres sectores de la Reserva Nacional Matsés, 2015.

\begin{tabular}{|c|c|c|c|c|}
\hline Orden / Especie & $\begin{array}{c}\text { Alemán } \\
\text { (238.1 km) }\end{array}$ & $\begin{array}{l}\text { Loboyacu } \\
\text { (214.5 km) }\end{array}$ & $\begin{array}{l}\text { Alto Gálvez } \\
(228.1 \mathrm{~km})\end{array}$ & Promedio \\
\hline Primates grandes & 25.58 & 14.08 & 26.64 & 22.12 \\
\hline Ateles chamek & 0.00 & 0.23 & 9.58 & 3.27 \\
\hline Lagothrix lagotricha & $23.53^{*}$ & $13.84^{*}$ & $15.03^{*}$ & 17.47 \\
\hline Alouatta seniculus & 1.05 & 0.00 & 2.02 & 1.022 \\
\hline Primates medianos & 15.45 & 20.07 & 31.10 & 2.21 \\
\hline Sapajus macrocephalus & 3.15 & 10.37 & 18.93 & 10.82 \\
\hline Cebus unicolor & 2.62 & 0.58 & 7.77 & 3.66 \\
\hline Pithecia monachus & $9.68^{*}$ & $9.11^{*}$ & 4.39 & 7.73 \\
\hline Primates pequeños & 35.00 & 30.14 & 49.14 & 38.09 \\
\hline Plecturocebus cupreus & 1.12 & 1.39 & 4.35 & 2.29 \\
\hline Saimiri macrodon & 2.80 & 0.00 & 26.75 & 9.85 \\
\hline Saguinus mystax & 20.58 & 17.87 & 13.22 & 17.22 \\
\hline Saguinus nigrifrons & 10.50 & 10.87 & 4.80 & 8.72 \\
\hline \multicolumn{5}{|l|}{ Perissodactyla } \\
\hline Tapirus terrestris & 0.10 & 0.23 & 0.33 & 0.22 \\
\hline \multicolumn{5}{|l|}{ Cetartiodactyla } \\
\hline Mazama spp. & 1.26 & 0.93 & 1.69 & 1.29 \\
\hline Paucituberculata & 1.89 & 3.38 & 10.40 & 5.22 \\
\hline Pecari tajacu & 1.89 & 3.38 & 4.39 & 3.22 \\
\hline Tayassu pecari & 0.00 & 0.00 & 6.01 & 2 \\
\hline Rodentia & 1.40 & 2.02 & 3.15 & 2.19 \\
\hline Dasyprocta fuliginosa & 1.40 & 1.86 & 3.15 & 2.14 \\
\hline Myoprocta pratti & 0.00 & 0.15 & 0.00 & 0.05 \\
\hline Carnivora & 4.72 & 0.11 & 7.32 & 4.05 \\
\hline Eira barbara & 0.31 & 0.11 & 0.33 & 0.25 \\
\hline Nasua nasua & 4.41 & 0.00 & 6.98 & 3.80 \\
\hline Pilosa & 0.10 & 0.11 & 0.33 & 0.18 \\
\hline Tamandua tetradactyla & 0.00 & 0.11 & 0.33 & 0.15 \\
\hline Choloepus didactylus & 0.10 & 0.00 & 0.00 & 0.03 \\
\hline Total de especies & 16 & 15 & 18 & \\
\hline
\end{tabular}

El resto de los grupos taxonómicos como ungulados, carnívoros, roedores y pilosos tuvieron densidades menores. No obstante, se resalta que $P$. tajacu fue más densa al comparar con estudios llevados a cabo en distintos lugares de la Amazonía peruana (Aquino et al., 2007a; Aquino et al., 1997; Bodmer et al., 1997; Lavajos \& Meza 2013; Aquino, 2007b; Pérez-Peña, 2011; Lleellish et al, 2003; Pérez-Peña et al., 2012; Aquino, 2015; Aquino et al., 2014). Esta diferencia podría estar relacionada a la preferencia de hábitat, P. tajacu prefiere hábitats más secos en las tierras altas (Bodmer 1997), los mismos que son predominantes en los tres sectores evaluados. Por otro lado, T. pecari se registró únicamente en bosques de terrazas bajas, ubicados adyacentemente al río Gálvez en el sector Alto Gálvez, cuya densidad fue menor a otros estudios realizados en áreas de baja presión de caza (Bodmer et al., 1997; Aquino 2015). Al parecer esta especie prefiere hábitats húmedos periódicamente inundables (Bodmer 1997; Aquino et al., 2007a). 
Las densidades de T. terrestris y Mazama spp. mantienen una tendencia poco variable en relación a trabajos realizados en otros lugares de la Amazonía peruana (Aquino et al., 2001; Hurtado \& Bodmer 2004; Aquino et al., 2007a; Aquino et al., 2007b; Pérez-Peña, 2011; Pérez-Peña et al., 2012). Esta tendencia puede deberse a características propias de estas especies, como la baja reproducción, vida solitaria y hábitos catemerales (hábito diurno y nocturno), el cual determinaría que sus poblaciones presenten densidades relativamente bajas y sean difíciles de observarlas en los muestreos diurnos (Brooks et al., 1999; Aquino et al., 2007a; Di Biteti et al., 2008; SERNANP, 2014b).

En términos generales en el sector del Alto Gálvez las densidades fueron mayores que en los demás sectores, la presencia de los tres primates grandes reportados para la zona como $A$. chamek, $L$. lagotricha y $A$. seniculus, confirman que estos ecosistemas se encuentran en buen estado de conservación. Incluso Cacajao calvus fue avistado en tres oportunidades de manera casual en bosques del rio Gálvez, de la cual se menciona que habita en bosques saludables (Aquino, 1997). Esto se explicaría gracias a que los cazadores llegan esporádicamente a este sector, a diferencia de los sectores Alemán y Loboyacu, donde la actividad antrópica es mayor, debido a que los anexos de las comunidades, se encuentran relativamente cerca a estos sectores y no requieren mucho tiempo y logística para realizar la cacería. Además, en el sector Alemán, según comentarios de algunos entrevistados, antes de la creación de la Reserva en el 2009 existía tala ilegal de madera, y que junto con la cacería indiscriminada, podrían haber provocado el desplazamiento y/o disminución de especies como Cacajao calvus y Ateles chamek que son más sensibles a perturbaciones (Pérez-Peña, 2011). Es decir, la sobrecaza de un lugar marca el patrón de presencia y abundancia de especies de grandes primates. Por ello, las zonas de Aleman y Loboyacu tuvieron poblaciones más reducidas en comparación al sector Gálvez, zona en mejor estado de conservación.

\section{CONCLUSIÓN}

En general los primates alcanzaron las densidades más altas, siendo Lagothrix lagotricha y Saguinus mystax abundantes en los tres sectores. El resto de grupos taxonómicos resultaron con menores densidades, de las cuales Tapirus terrestris y Eira barbara fueron las especies menos densas. Asimismo, se resalta que la comunidad de

\section{PCA Plot - Covariance - ACPAAIIAP}

Vector - Axis 1

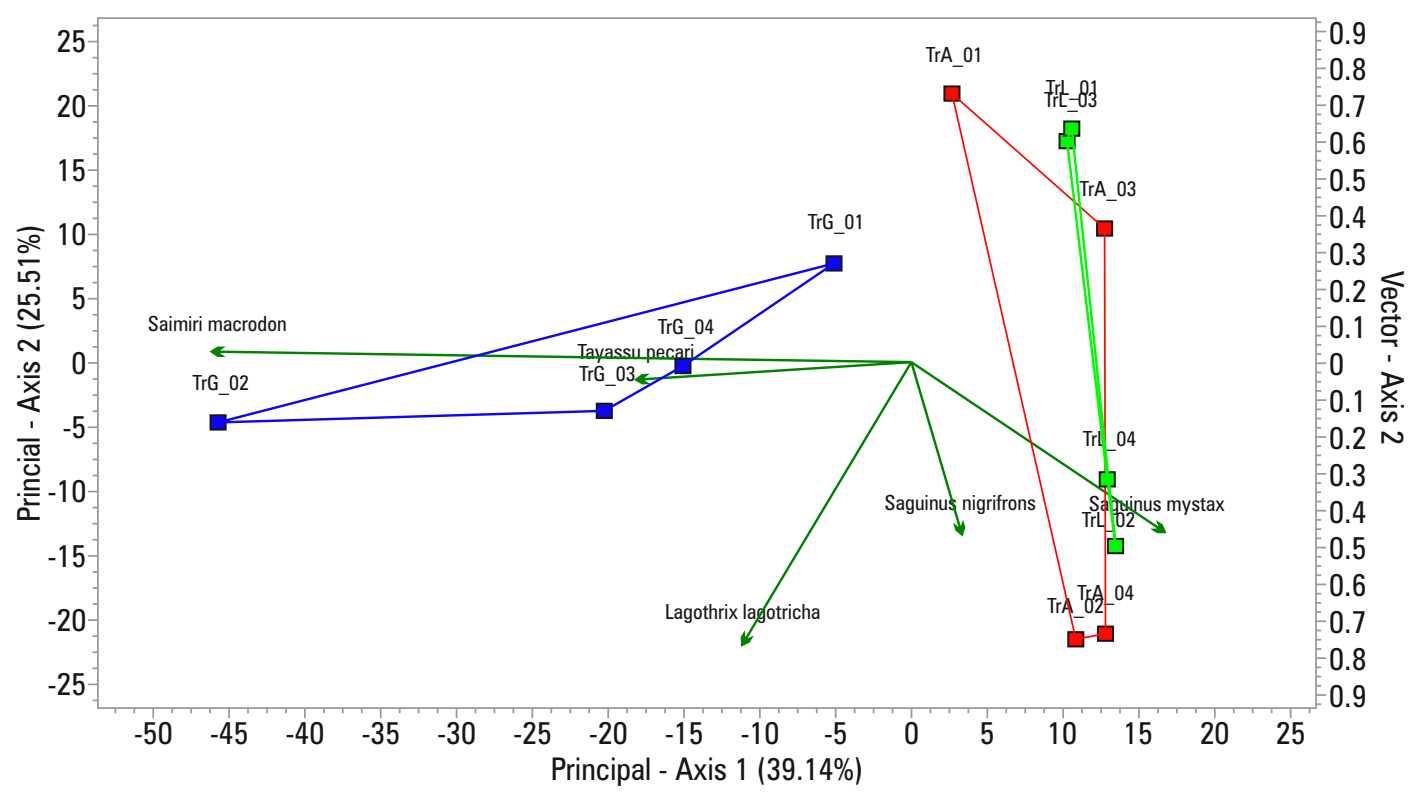

Figura 2. Análisis de componentes principales usando matriz de covarianza en la densidad de mamíferos de caza entre los sectores. 
mamíferos del sector Alemán y Loboyacu son diferentes al sector Alto Gálvez, en donde las densidades son más altas. Además la presencia de los tres primates grandes en este sector, demuestra el buen estado de conservación de sus bosques.

\section{AGRADECIMIENTO}

Con especial consideración y estima a Nidya Carola Carpio Martínez, Jefe de la Reserva Nacional Matsés-SERNANP, los guardaparques Caled Rodríguez López, Elmer Antonio Icomena Ahuanari, Teodoro Dunu Sedenteyuque, Daniel Mozombite Trejo, Richart Reyna López, Wilmer Bina Moconoqui y Glen Manuyama Rengifo; los comuneros Juler Samuel Zumba Oroche, Atilio Reyna López, Juvencio Reyna López, Antonio Reyna, Esteban Ahuanari Gonzáles, Leoncio Braga Huaycama, Juan Pablo Macaya Mori, por su amistad y valioso trabajo como guías de campo. A Carlos Vásquez Salas, Marco Miguel Odicio Iglesias y Pedro E. Pérez Peña por su apoyo técnico.

\section{REFERENCIAS BIBLIOGRÁFICAS}

Aquino, R. 1997. Bases ecológicas y alternativas para la conservación del huapo rojo Cacajao calvus ucayali. En: Fang, T.; Montenegro, O.; Bodmer, R. Manejo (Eds). Conservación de fauna silvestre en América Latina. La PazBolivia: Instituto de Ecología. p. 290-301.

Aquino, R.; Bodmer, R.E.; Etersit, P. 1997. Evaluación de poblaciones del pecari de collar (Tayassu tajacu) y pecari labiado (T. pecari) en la cuenca del río Pucacuro, río Alto Tigre. En: Fang, T.; Montenegro, O.; Bodmer, R. Manejo (Eds) Conservación de fauna silvestre en América Latina. La Paz-Bolivia: Instituto de Ecología. p. 469-478.

Aquino, R.; Bodmer, R.E.; Gil, JG. 2001. Mamiferos de la cuenca del río Samiria: Ecología poblacional y sustentabilidad de la caza. Lima. $141 \mathrm{pp}$.

Aquino, R.; Pacheco, T.; Vásquez, M. 2007a. Evaluación y valorización económica de la fauna silvestre en el río Algodón, Amazonía peruana. Revista Peruana de Biología, 14 (2): 187-192.

Aquino, R.; Terrones, C.; Navarro, R.; Terrones, W. 2007b. Evaluación del impacto de la caza en mamíferos de la cuenca del río Alto Itaya, Amazonía peruana. Revista Peruana de Biología, 14 (2): 181-186.

Aquino, R.; García, G.; Charpentier, E. 2014. Abundancia de ungulados y uso de hábitats entre los ríos Bajo Urubamba y Tambo, Amazonía peruana. Ciencia Amazónica, 4(1):13-21.
Aquino, R.E.; López, L.; Arévalo, I.; García, G.; Charpentier, E. 2015. Densidad de ungulados en bosques de baja y alta presión de caza en el nororiente de la Amazonía peruana. Ciencia Amazónica, 4(2): 128-137.

Bodmer, R.; Aquino, R.; Puertas, P.; Reyes, C.; Fang T.G.; Gottdenker, N. 1997. Manejo y uso sustentable de pecaries en la Amazonía peruana. Unión Internacional para la Conservación de la Naturaleza, Quito. 112pp.

Bordmer, R.E.; Fang, T.G.; Puertas, P.E. Antúnez, M.; Chota, K.; Bodmer, W.E. 2014. Cambio climático y fauna silvestre en la Amazonía peruana: Impacto de la sequía e inundaciones en la Reserva Nacional Pacaya Samiria. Fundación Latinoamericana para el Trópico Amazónico. Iquitos. 256pp.

Brooks, D.M.; Eisenberg, J.F. 1999. Estado y Biología de los Tapires Neotropicales: Perspectiva General. En: Fang, T.; Montenegro, O.; Bodmer, R. Manejo (Eds) Conservación de fauna silvestre en América Latina. La PazBolivia: Instituto de Ecología. p. 409-414.

Burnham, K.P.; Anderson, D.R.; Laake, J.L. 1980. Estimation of Density from Line Transect Sampling of Biological Populations. Wildlife Monographs, 72: 202pp.

Hurtado-Gonzales, J.L.; Bodmer, R.E. 2004. Assessing the sustainability of brocket deer hunting in the Tamshiyacu-Tahuayo Communal Reserve, Northeastern Peru. Biological Conservation, 116: 1-7.

Lavajos, L.E.; Meza, D.W. 2013. Estado actual de las poblaciones de mamíferos y aves de caza utilizados por las comunidades del Área de Conservación Regional Comunal TamshiyacuTahuayo, Loreto. Tesis de biólogo, Universidad Nacional de la Amazonía Peruana. Loreto, Perú.

Lleellish, M.; Amanzo, J.; Hooker, Y.; Yale, S. 2003. Evaluación poblacional de pecaríes en la región del Alto Purús. En: Leite, R.; Pitman, N.; Alvaréz, Patricia (Eds). Alto Purús biodiversidad conservación y manejo. p. 123135.

MINAM. 2009. Decreto Supremo N N 014-2009MINAM.

Pérez-Peña, P. 2011. Evaluación poblacional de mamíferos y aves de caza en la Concesión de Conservación Lago Preto Paredón y en cuenca media del Yavarí Mirín. Reporte final. Iquitos. $31 \mathrm{pp}$.

Pérez-Peña, P.E.; Ruck, L.; Riveros, M.S.; Rojas, G. 2012. Evaluación del conocimiento indígena Kichwa como herramienta de monitoreo en la abundancia de animales de caza. Folia Amazónica, 21(1): 115-127. 
Pezo, E. 2007. Bases bio-ecológicas para una estrategia de conservación de Tapirus terrestris (Linneaus 1758) en las Reserva Pacaya-Samiria y Reserva Comunal Tamshiyacu-Tahuayo. Tesis de maestría, Universidad Nacional de la Amazonía Peruana, Facultad de Ciencias Biológicas, Iquitos, Perú.

Puertas, P.E.; Pinedo, A.; Soplin, S.; Antúnez, M.; López, L.; Caro, J.; Chicaje, L.; Panduro, R.; Vásquez, R.; Flores, J.L. 2017. Evaluación poblacional y uso sostenible de animales de caza por comunidades indígenas en el Area de Conservación Regional Ampiyacu Apayacu, Noreste de la Amazonía peruana. Folia Amazónica, 26(1): 37-50.

Rosin, C.; Swamy, V. 2013. Vaiable density responses of primate communities to hunting pressure in a western Amazonian river basin. Neotropical Primates 20 (1): 25-31.

Rumiz, D.I. 2010. Roles ecológicos de los mamíferos medianos y grandes. En: Wallace, R.B.; Gómez, H.; Porcel, Z.R.; Rumiz, D.I. (Eds). Distribución, ecología y conservación de los mamiferos medianos y grandes de Bolivia. $\mathrm{p}$. 53-73.
SERNANP. 2014a. Plan Maestro de la Reserva Nacional Matsés 2014-2019. Servicio Nacional de Áreas Naturales Protegidas, Lima. 94pp.

SERNANP. 2014b. Plan de manejo de la Reserva Nacional Pucacuro, realizado por cazadores kichwas de la Comunidad 28 de Julio, Alfonso Ugarte y Asociación de Cazadores de Intuto 2014-2018. Servicio Nacional de Áreas Naturales Protegidas, Lima. 43pp.

Tafur, M.P. 2010. Evaluación de la sostenibilidad de la cacería de mamíferos en la Comunidad de Zancudo, Reserva Nacional Natural Puinawai, Guainía-Colombia. Tesis de maestría, Universidad Nacional de Colombia, Facultad de Ciencias, Bogotá, Colombia. 101pp.

Vriesendorp, C.; Pitman, N.; Rojas, J.I.; Pawlak, B.A.; Calixto, L.; Vela, M.; Fasabi, P. 2006. Matses Rapid Biological Inventories Report 16. The Field Museum. Chicago, Illinois.

Recibido: 27 de Julio del 2017

Aceptado para publicación:21 de Agosto del 2017 